\title{
ROLLING OF ELASTIC CYLINDERS WITH FRICTION AT SUPERSONIC SPEED
}

\author{
J. R. BARBER \\ Dept. of Mechanical Engineering and Applied Mechanics, University of Michigan, Aan Arbor, MI 48109 , \\ U.S.A. \\ and \\ Maria Comminou \\ Dept. of Civil Engineering, University of Michipan, Ann Arbor, MI 48109, U.S.A.
}

(Received 24 August 1981)

\begin{abstract}
Abtract-A closed form solution is given for the supersonic rolling of an elastic cylinder on a plane using the method of the preceding paper[1]. Tangential and normal forces are transmitted and Coulomb friction is assumed.
\end{abstract}

\section{INTRODUCTION}

In the preceding paper[1], we gave a general solution for the contact tractions transmitted between two elastic half-spaces when a disturbance propagates along their boundary at supersonic speed. This problem originally arose in the context of the interaction of a plane elastic pulse or wave with a unilateral interface [2-5], but a disturbance propagating along the interface can also be produced by purely kinematic means if the bodies are slightly curved and roll over each other. This is the subject of the present paper.

\section{STATEMENT OF THE PROBLEM}

We consider the problem of an elastic cylinder of radius $R$ rolling with friction on an elastic half-space with velocity $v$ which is supersonic with respect to the materials of the two bodies. The corresponding quasi-static problem has been treated by Carter [6] for similar materials and Bentall and Johnson[7] for dissimilar materials. Craggs and Roberts [8] discuss the supersonic rolling problem for a frictionless cylinder.

The bodies are pressed together by a compressive force $P$ and transmit a tangential force $Q$ and torque $Q R$ as shown in Fig. 1. In the steady-state, the disturbance is stationary with respect to a co-ordinate system moving at velocity $v$, and plane $P$ and $S V$ pulses will be generated in both solids at angles given by eqns (1) and (2) of [1]. In the present example, there is no incident pulse, but we can define a dimensionless co-ordinate through

$$
\eta=\left(x_{1}-v t\right) / R
$$

Comparing this equation with eqn (3) of [1], we see that it is formally equivalent to writing

$$
k_{0} \sin \theta_{0}=1 / R
$$

and the same substitution in subsequent equations of [1] will suffice to cast them in the correct form for the present problem.

\section{KINEMATICS}

In general, we anticipate that the angular velocity $\Omega$ (clockwise positive) will differ from $v / R$ and hence that some global slip $u$ will occur at the contact region, where

$$
\boldsymbol{u}=\boldsymbol{v}-\boldsymbol{R} \Omega \text {. }
$$

Note that global slip in rolling contact is a consequence of elastic deformation and does not depend on the presence of slip zones, nor even on the force $Q$ being non-zero (see for example, 


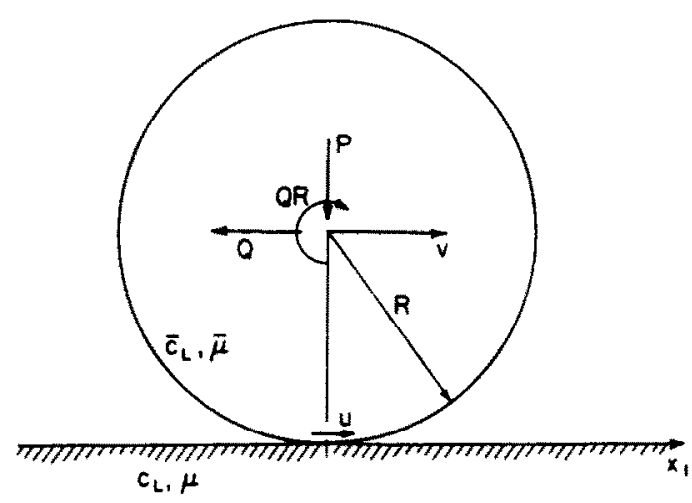

Fig. 1. The cylinder rolling on a plane.

Spence [9]). We define the slip ratio

$$
U=u l v
$$

To use the results of [1], we first determine the gap opening and slip velocities, $G_{0}(\eta), H_{0}(\eta)$ respectively, which would occur if there were no tractions transmitted between the bodies. We find

$$
\begin{gathered}
G_{0}(\eta)=-v \eta \\
H_{0}(\eta)=u
\end{gathered}
$$

where we have assumed that $U, \eta$ are small compared with unity as required by the small strain theory of elasticity.

\section{SOLUTION}

The solution is obtained as in [1], to which the reader is referred for more detailed explanation and definition of notation. We use eqns (6) and (7) to plot a diagram illustrating the relative values of $\gamma_{1} G_{0}(\eta), \gamma_{2} G_{0}(\eta), H_{0}$ sgn $\lambda_{1}$ and hence determine the extent of the slip and stick zones. We restrict attention to the more common case $\gamma_{1}>0$, but consider separately the cases $\gamma_{2}>0, \gamma_{2}<0$ which give qualitatively different results.

$\gamma_{2}<0$

The appropriate diagram for $\gamma_{2}<0$ and $u$ sgn $\lambda_{1}>0$ is shown in Fig. 2(a), corresponding to the free surface condition of Fig. 2(b). The diagram is shaded in accordance with the inequalities in [1] which define the conditions for stick and slip. For example, stick is only permitted in the range $\gamma_{2} G_{0}>H_{0} \operatorname{sgn} \lambda_{1}>\gamma_{1} G_{0}$ and hence can only occur between the two lines $-\gamma_{2} v \eta,-\gamma_{1} v \eta$ in the right part of Fig. 2(a) as shown.

Contact will start at the point $A$, where the free surface solution predicts interpenetration (see Fig. $2 b$ ) and extend to the left until $B$, beyond which only separation is possible.

For the case illustrated, the entire contact zone must be in a state of conforming slip and the tractions are

$$
\begin{gathered}
N=\frac{-\mu v \eta}{c_{L}\left(\lambda_{2}+f\left|\lambda_{1}\right|\right)} \\
S \operatorname{sgn} \lambda_{1}=\frac{\mu v f \eta}{c_{L}\left(\lambda_{2}+f\left|\lambda_{1}\right|\right)}
\end{gathered}
$$

from eqns (30) and (31) of [1] and eqn (6).

Suppose we now increase the depth of penetration, $d$, in Fig. 2(b), so that the point $A$ falls in 


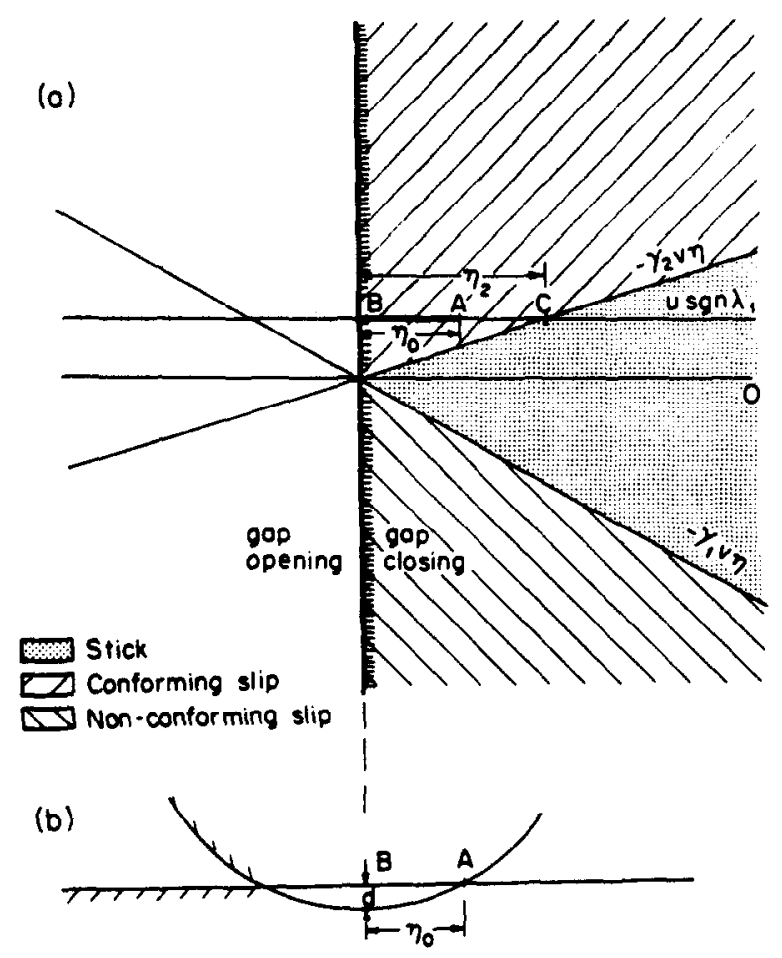

Fig. 2. (a) Graphical determination of slip and stick zones, $\gamma_{1}>0, \gamma_{2}<0$. (b) The free surface geometry.

the stick region to the right of $C$ in Fig. 2(a). The contact will now contain a conforming slip zone $0<\eta<\eta_{2}$ in which the tractions are still given by (8) and (9) above and a stick zone $\eta_{2} \leq \eta<\eta_{0}$ in which

$$
\begin{gathered}
N=\frac{\mu\left(\left|\lambda_{1}\right| u \operatorname{sgn} \lambda_{1}-\lambda_{3} v \eta\right)}{c_{L}\left(\lambda_{1}{ }^{2}+\lambda_{2} \lambda_{3}\right)} \\
S \operatorname{sgn} \lambda_{1}=\frac{\mu\left(\lambda_{2} u \operatorname{sgn} \lambda_{1}+\left|\lambda_{1}\right| v \eta\right)}{c_{L}\left(\lambda_{1}{ }^{2}+\lambda_{2} \lambda_{3}\right)}
\end{gathered}
$$

from eqns (16) and (17) of [1] and (6) and (7). The stick slip boundary is given by

$$
\eta_{2}=-\frac{U \operatorname{sgn} \lambda_{1}}{\gamma_{2}}
$$

The force resultants $P, Q$, transmitted by the contact zone can be found by integrating eqns (8)-(11). Thus,

$$
\begin{aligned}
& P=-R \int_{0}^{\eta_{0}} N(\eta) \mathrm{d} \eta \\
& Q=-R \int_{0}^{\eta_{0}} S(\eta) \mathrm{d} \eta
\end{aligned}
$$

from which

$$
\begin{array}{rlrl}
\frac{P}{\mu R} \frac{c_{L}}{v} & =\frac{\eta_{0}^{2}}{2\left(\lambda_{2}+f\left|\lambda_{1}\right|\right)} ; & 0<\eta_{0}<\eta_{2} \\
& =\frac{\lambda_{3} \eta_{0}^{2}+\left|\lambda_{1}\right| \gamma_{2} \eta_{2}\left(2 \eta_{0}-\eta_{2}\right)}{2\left(\lambda_{1}^{2}+\lambda_{2} \lambda_{3}\right)} ; \quad \eta_{0}>\eta_{2}
\end{array}
$$




$$
\begin{aligned}
\frac{Q \operatorname{sgn} \lambda_{1}}{\mu R} \frac{c_{L}}{v} & =\frac{-f \eta_{0}^{2}}{2\left(\lambda_{2}+f\left|\lambda_{1}\right|\right)} ; \quad 0<\eta_{0}<\eta_{2} \\
& =\frac{-\left|\lambda_{1}\right| \eta_{0}^{2}+\lambda_{2} \gamma_{2} \eta_{2}\left(2 \eta_{0}-\eta_{2}\right)}{2\left(\lambda_{1}^{2}+\lambda_{2} \lambda_{3}\right)} ; \quad \eta_{0}>\eta_{2} .
\end{aligned}
$$

Notice that the ratio $c_{2} / v$ which appears in these equations is equal to $\sin \theta_{1}$ where $\theta_{1}$ is the angle of reflection of $\boldsymbol{P}$ pulses from the moving disturbance. The same factor appears in the definition of the $\lambda_{i}$ (eqns (9)-(11) of [1]) and it will cancel if the equations are expanded in terms of the material constants.

An exactly parallel analysis can be conducted for the case $u \operatorname{sgn} \lambda_{1}<0$. The line $B A C$ now falls in the lower part of Fig. 2(a).

If $\eta_{0}<\eta_{1}$, where

$$
\eta_{1}=-\frac{U \operatorname{sgn} \lambda_{1}}{\gamma_{1}}
$$

we have non-conforming slip only and the tractions are

$$
\begin{aligned}
N & =\frac{-\mu v \eta}{c_{L}\left(\lambda_{2}-\left|\lambda_{1}\right| f\right)} \\
S \operatorname{sgn} \lambda_{1} & =\frac{-\mu v f \eta}{c_{L}\left(\lambda_{2}-\left|\lambda_{1}\right| f\right)} .
\end{aligned}
$$

If $\eta_{0}>\eta_{1}$, stick will occur in the range $\eta_{0}>\eta>\eta_{1}$ where the tractions are given by eqns (10) and (11) above.

The resultant forces are

$$
\begin{aligned}
\frac{P}{\mu R} \frac{c_{L}}{v} & =\frac{\eta_{0}^{2}}{2\left(\lambda_{2}-f\left|\lambda_{1}\right|\right)} ; & & 0<\eta_{0}<\eta_{1} \\
& =\frac{\lambda_{3} \eta_{0}{ }^{2}+\left|\lambda_{1}\right| \gamma_{1} \eta_{1}\left(2 \eta_{0}-\eta_{1}\right)}{2\left(\lambda_{1}{ }^{2}+\lambda_{2} \lambda_{3}\right)} ; & & \eta_{0}>\eta_{1} \\
\frac{Q \operatorname{sgn} \lambda_{1}}{\mu R} \frac{c_{L}}{v} & =\frac{f \eta_{0}{ }^{2}}{2\left(\lambda_{2}-f\left|\lambda_{1}\right|\right)} ; & & 0<\eta_{0}<\eta_{1} \\
& =\frac{-\left|\lambda_{1}\right| \eta_{0}{ }^{2}+\lambda_{2} \gamma_{1} \eta_{1}\left(2 \eta_{0}-\eta_{1}\right)}{2\left(\lambda_{1}{ }^{2}+\lambda_{2} \lambda_{3}\right)} ; & & \eta_{0}>\eta_{1} .
\end{aligned}
$$

\section{SOLUTION WHEN $P, Q$ ARE PRESCRIBED}

The ratio of tangential to normal force must be in the range $f \geq Q \operatorname{sgn} \lambda_{1} / P \geq-f$. It takes the upper limit when there is non-conforming slip only ( $u \operatorname{sgn} \lambda_{1}<0, \eta<\eta_{1}$, eqns (21) and (23)) and the lower limit when there is conforming slip only ( $u \operatorname{sgn} \lambda_{1}>0, \eta<\eta_{2}$, eqns (15) and (17)).

If the value of $Q \operatorname{sgn} \lambda_{1} / P$ is between these limits, we must have stick and either conforming or non-conforming slip. With conforming slip and stick

$$
\frac{Q \operatorname{sgn} \lambda_{1}}{P}=\frac{-\gamma_{2}\left|\lambda_{1}\right|-\lambda_{2}\left\{2 \gamma_{2}\left(\frac{U \operatorname{sgn} \lambda_{1}}{\eta_{0}}\right)+\left(\frac{U}{\eta_{0}}\right)^{2}\right\}}{\gamma_{2} \lambda_{3}-\left|\lambda_{1}\right|\left\{2 \gamma_{2}\left(\frac{U \operatorname{sgn} \lambda_{1}}{\eta_{0}}\right)+\left(\frac{U}{\eta_{0}}\right)^{2}\right\}} ; \quad \eta_{0}>\eta_{2} ; U \operatorname{sgn} \lambda_{1}>0
$$


from eqns (16) and (18), while for non-conforming slip and stick

$$
\frac{Q \operatorname{sgn} \lambda_{1}}{P}=\frac{-\gamma_{1}\left|\lambda_{1}\right|-\lambda_{2}\left\{2 \gamma_{1}\left(\frac{U \operatorname{sgn} \lambda_{1}}{\eta_{0}}\right)+\left(\frac{U}{\eta_{0}}\right)^{2}\right\}}{\gamma_{1} \lambda_{3}-\left|\lambda_{1}\right|\left\{2 \gamma_{1}\left(\frac{U \operatorname{sgn} \lambda_{1}}{\eta_{0}}\right)+\left(\frac{U}{\eta_{0}}\right)^{2}\right\}} \quad \eta_{0}>\eta_{1} ; U \operatorname{sgn} \lambda_{1}<0
$$

from (23) and (25). Thus $Q \operatorname{sgn} \lambda_{1} / P$ depends on $\eta_{0}, U$ only through the ratio $U \operatorname{sgn} \lambda_{1} / \eta_{0}$ and it follows from eqns (26) and (27) that conforming slip and stick will occur if

$$
-f<\frac{Q \operatorname{sgn} \lambda_{1}}{P}<\frac{-\left|\lambda_{1}\right|}{\lambda_{3}}
$$

while non-conforming slip and stick occurs if

$$
\frac{-\left|\lambda_{1}\right|}{\lambda_{3}}<\frac{Q \operatorname{sgn} \lambda_{1}}{P}<+f
$$

Thus if $P, Q$ are prescribed we can determine which regime will occur from (28) and (29) and then solve for $\eta_{0}, U$ from the appropriate equations (16) and (18) or (23) and (25).

Figure 3(a) shows the total contact length $\eta_{0}$ and its division into stick and slip for the representative case $\left|\lambda_{1}\right|=0.2, \lambda_{2}=1, \lambda_{3}=1, f=0.5$. Complete stick occurs when $Q \operatorname{sgn} \lambda_{1} / P=$ $-\left|\lambda_{1}\right| \mid \lambda_{3}$. We note that $\eta_{0}$ is comparatively insensitive to variation in $Q$ at given $P$. Corresponding results for the slip ratio $U$ are given in Fig. 3(b).

A special case of some interest is that of similar materials for which $\left|\lambda_{1}\right|$ is zero and hence

$$
\gamma_{1}=-\gamma_{2}=\lambda_{3} f / \lambda_{2}
$$

The lines $-\gamma_{1} v \eta,-\gamma_{2} v \eta$ in Fig. 2(a) then become symmetric about the horizontal axis and Figs. $(3 a, b)$ become symmetric and anti-symmetric respectively about $Q \operatorname{sgn} \lambda_{1} / P=0$.

All the above discussion applies to the case $\gamma_{2}<0$ for which $\left|\lambda_{1}\right| \mid \lambda_{3}<f$. However, it can be seen from Fig. 3(a) or the inequality (28) that as $\left|\lambda_{1}\right| \mid \lambda_{3}$ approaches $f$ the range of values of $Q$ sgn $\lambda_{1} / P$ for which conforming slip and stick can occur diminishes.

$\gamma_{2}>0$

We now consider the case $\left|\lambda_{1}\right|>f \lambda_{3}$ and hence $\gamma_{2}>0$. The permissible domains for stick and slip are shown in Fig. 4(a) and we see immediately that stick can only occur if $u \operatorname{sgn} \lambda_{1}<0$.

If $\eta_{0}<\eta_{2}$, i.e. if contact does not extend to the right of $D$ in Fig. $4(a)$, the previous analysis still applies and the tractions and transmitted forces are given by eqns (20)-(25).

For $\eta_{0}>\eta_{2}$, three contact zones are developed: non-conforming slip $0<\eta<\eta_{1}$; stick in $\eta_{1}<\eta<\eta_{2}$ and conforming slip $\eta_{2}<\eta<\eta_{0}$. The tractions in the first two zones are unchanged from the case $\eta_{0}<\eta_{2}$ and in the conforming slip zone they are given by eqns (8) and (9). Hence, we can calculate the transmitted forces which are

$$
\begin{gathered}
\frac{P}{\mu R} \frac{c_{L}}{v}=\frac{\eta_{0}{ }^{2}}{2\left(\lambda_{2}+f\left|\lambda_{1}\right|\right)}+\frac{\left|\lambda_{1}\right|\left(\gamma_{1}-\gamma_{2}\right) \eta_{1} \eta_{2}}{2\left(\lambda_{1}{ }^{2}+\lambda_{2} \lambda_{3}\right)} \\
\frac{Q \operatorname{sgn} \lambda_{1}}{\mu R} \frac{c_{L}}{v}=\frac{-f \eta_{0}{ }^{2}}{2\left(\lambda_{2}+f\left|\lambda_{1}\right|\right)}+\frac{\lambda_{2}\left(\gamma_{1}-\gamma_{2}\right) \eta_{1} \eta_{2}}{2\left(\lambda_{1}{ }^{2}+\lambda_{2} \lambda_{3}\right)}
\end{gathered}
$$

The variation of $\eta_{0}, U \operatorname{sgn} \lambda_{1}$ with $Q \operatorname{sgn} \lambda_{1} / P$ is shown in Figs. $5(\mathrm{a}, \mathrm{b})$ for the same values as Fig. 3 except that $f=0.1$. As $Q \operatorname{sgn} \lambda_{1} / P$ decreases from $+f$, the stick zone increases until a conforming slip zone develops at the leading edge. This zone then grows at the expense of the non-conforming slip and stick zones whose lengths remain in the fixed ratio $\gamma_{2} /\left(\gamma_{1}-\gamma_{2}\right)$ until there is conforming slip everywhere at $Q \operatorname{sgn} \lambda_{1} / P=-f$. 

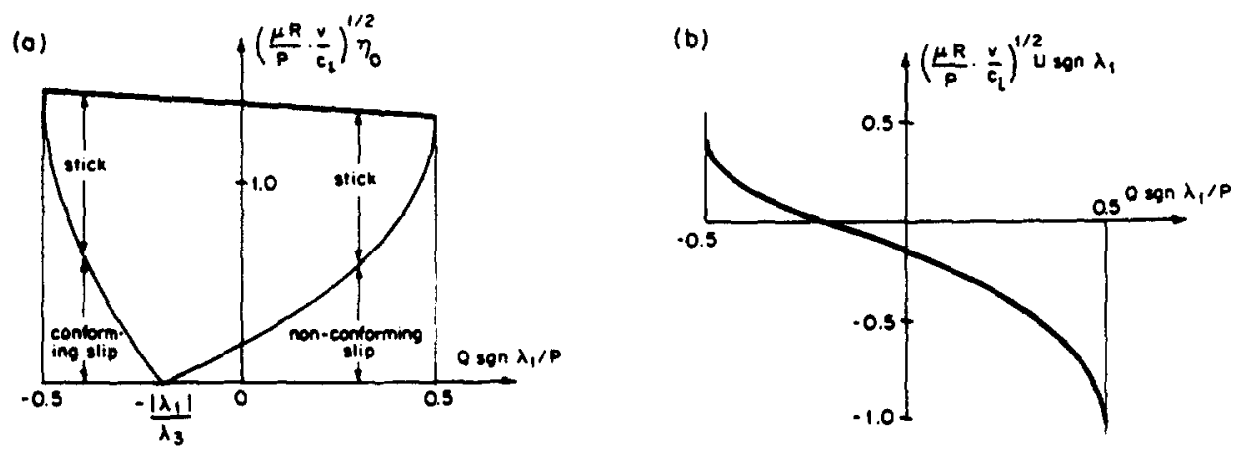

Fis. 3. Effect of the applied loads $P, Q$ when $\eta_{2}<0$ on (a) the extent of the contact repion $\eta_{0}$ and its division into stick and slip zones. (b) The slip ratio $U$.

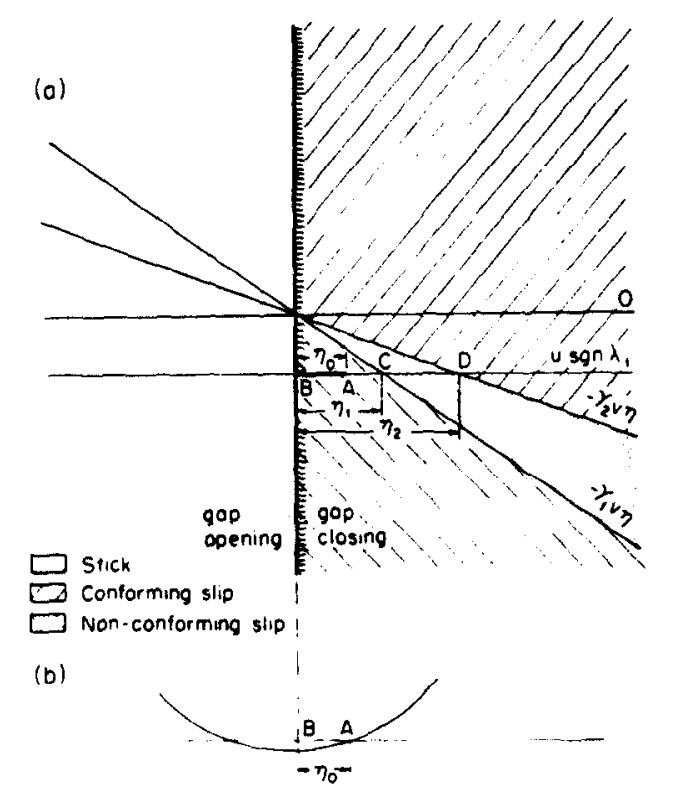

Fig. 4. (a) Graphical determination of slip and stick zones, $y_{1}>0, \gamma_{2}>0$. (b) The free surface geometry. 

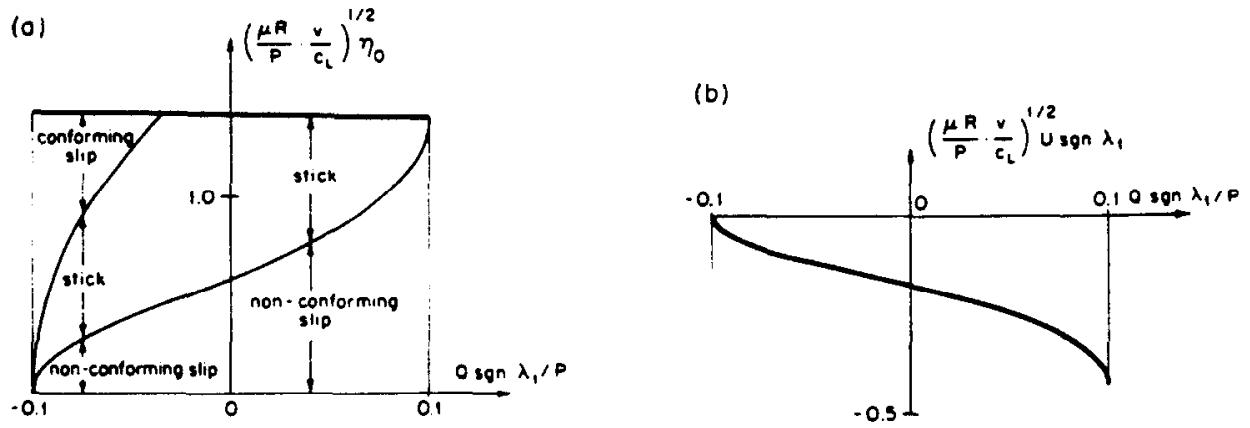

Fig. S. Effect of the applied loads $P, Q$ when $\eta_{2}>0$ on (a) the extent of the contact region $\eta_{0}$ and its division into stick and slip zones. (b) The slip ratio $U$.

Acknowledgement-Support by the National Science Foundation through the grant CME 8019281 gratefully acknowledged.

\section{REFERENCES}

1. J. R. Barber, M. Comninou and J. Dundurs, Contact transmission of wave motion between two solids with an initial gap. Int. J. Solids Structures, 18, 775 (1982).

2. M. Comninou and J. Dundurs, Reflection and refraction of elastic waves in presence of separation. Proc. R. Soc. A3s6, 509.

3. M. Comninou and J. Dundurs, Interaction of elastic waves with a unilateral interface. Proc. R. Soc. A36, 141 (1979).

4. J. Dundurs and M. Comninou, Interface separation caused by a plane elastic wave of arbitrary form. Wave Motion 1, 17 (1979).

5. M. Comninou, J. R. Barber and J. Dundurs, Disturbance at a frictional interface caused by a plane elastic pulse. J. Appl. Mech, in press.

6. R. H. Bentall and K. L. Johnson, Stip in the rolling contact of two dissimilar elastic rollers. Int. J. Mech. Sci. 9, 389 (1967).

7. F. C. Carter, On the action of a locomotive driving wheel. Proc. R. Soc. A112, 151 (1926).

8. J. W. Craggs and A. M. Roberts, On the motion of a heavy cylinder over the surface of an elastic solid. J. Appl. Mech. 34. 207 (1967).

9. D. A. Spence. Self similar solutions to adhesive contact problems with incremental loading. Proc. R. Soc. A305, 55 (1968). 